OPEN ACCESS

Edited by:

Petra Granitzer,

University of Graz, Austria

Reviewed by:

lam Choon Khoo,

Pennsylvania State University,

United States

Andrey Miroshnichenko,

University of New South Wales,

Australia

*Correspondence:

Yuancheng Fan

phyfan@nwpu.edu.cn

Fuli Zhang

fuli.zhang@nwpu.edu.cn

Specialty section:

This article was submitted to

Optics and Photonics,

a section of the journal

Frontiers in Physics

Received: 24 November 2020

Accepted: 25 January 2021

Published: 24 February 2021

Citation:

Xu J, Yang R, Fan Y, Fu Q and Zhang F

(2021) A Review of Tunable

Electromagnetic Metamaterials With

Anisotropic Liquid Crystals.

Front. Phys. 9:633104.

doi: 10.3389/fphy.2021.633104

\section{A Review of Tunable Electromagnetic Metamaterials With Anisotropic Liquid Crystals}

\author{
Jing Xu, Ruisheng Yang, Yuancheng Fan *, Quanhong Fu and Fuli Zhang * \\ Key Laboratory of Light-Field Manipulation and Information Acquisition, Ministry of Industry and Information Technology and
} School of Physical Science and Technology, Northwestern Polytechnical University, Xi'an, China

The performance of metamaterial is limited to a designed narrow band due to its resonant nature, it is highly desirable to incorporate active inclusions in metamaterials to extend the operation bandwidth. This review summarizes the development in realizing the tunability of electromagnetic response in metamaterials incorporated with nematic liquid crystal (LC). From rigorous comparison, it is found that the anisotropic property of nematic LC is essential in predicting the influence of LC molecular director orientation on the resonant frequency of metamaterials. By carefully designing the metamaterials and properly infiltrating LC, the operation frequency of single/double negative parameters of metamaterials can be dynamically modulated with remarkable red/blue-shift, depending on the LC molecular orientation angle. Moreover, the recent liquid crystalbased developments and novel applications are investigated and highlighted.

Keywords: tunable metamaterials, negative index metamaterial, liquid crystals, Liquid crystals reorientations, reconfigurable metamaterials

\section{INTRODUCTION}

In most cases, the electromagnetic parameters of natural materials are positive and larger than unity, while the materials whose optical parameters are less than unity or even negative are only investigated in specific hypotheses. Recently, metamaterials with customizable performance have attracted a great deal of attention in the fields of optics, electronics, physics, and materials, since their optical properties can be arbitrarily tailored by rationally designing their micro/nanostructures. Theoretically, the electromagnetic parameters of metamaterials (including the permittivity, permeability, and refractive index) can be designed with any desired values (even to negative), which provides an ideal platform for the free manipulation of electromagnetic waves.

In 1968, Veselago theoretically proposed a medium with both negative permittivity and permeability for the first time [1]. According to a rigorous analytical investigation, such a medium can exhibit various intriguing properties such as negative index, reversed Doppler effects, and perfect lens, etc. [2-5]. In natural materials, ferrite can exhibit negative permeability around its resonant frequencies, while metal can exhibit negative permittivity below its electron plasmon frequency [6]. However, research related to negative electromagnetic parameters has seldom been reported for a long time since natural materials rarely exhibit such special properties. To broaden the design strategies of material parameters, in 1996, Pendry proposed a kind of artificial structure with periodic split ring resonators (SRR), to significantly enhance the magnetic resonance and realize negative effective permeability [7]. Moreover, Pendry also designed a thin wire array to lower the plasma frequency and obtain a limited negative permittivity in a microwave frequency band [8]. Inspired by Pendry's works, D. R. Smith combined periodic metallic SRR and a wire array 
together to promote simultaneous negative permittivity and permeability in a certain frequency band, and thus realized a negative refractive index for the first time in experiment [9]. They found that periodic SRR exhibited an effective negative permeability, and the wire array alone exhibited a negative permittivity below its effective plasma frequency, while with the SRR and wire array together, a passband would appear when both permittivity and permeability were negative. The experimental demonstration of negative index along with a serial confirmation of extraordinary behaviors has attracted increased interest for studies on metamaterials [10-12]. During the last few decades, metamaterials with various different structural designs including Omega [13], S type [14], and fishnet $[15,16]$, have been proposed. The rapid development of metamaterials has also stimulated many promising applications such as diffraction-unlimited imaging [17-19], invisible cloaks $[20,21]$, subwavelength high-Q cavities [22-25], polarization manipulation [26], and electromagnetic absorbers [27-30]. It is significant that the freely designable electromagnetic parameters of metamaterials are important for the implementation of such devices.

Metamaterials have shown exterior capability to manipulate electromagnetic waves, but there are still some problems hindering their development toward practical applications. As for traditional metamaterials, once the structural units are determined, they can only work well at a certain frequency range, which greatly limits their applications in practical engineering. The ever-increasing demand on designing versatile photonic devices inspires the rapid development of tunable metamaterials [31-35]. Several methods have been proposed to broaden their operation bandwidth with the incorporation of active inclusions, such as varactor diodes [36-38], semiconductors [39-41], ferroelectric [42], phase change materials [43], graphene [44-46], and anisotropic materials [47-50].

Liquid crystal (LC) has emerged as a promising candidate for the manipulation of light waves since its director axis and optical properties are strongly dependent on the surface effect and ambient temperature, and its optical properties are very sensitive to the variation of external fields (such as the electric field, magnetic field, light field, sound field, etc.) $[51,52]$. The anisotropy of LC covers a wide frequency range from ultraviolet to microwave. Moreover, LC-based devices have many advantages such as small size, light weight, low cost, simple process, and continuous tunability. Therefore, as an excellent tunable functional material, LC is widely used in the optoelectronic fields. Recently, tunable metamaterials incorporated with liquid crystals have been widely reported in microwave, terahertz (THz), and even infrared bands. LC-based tunable metamaterials taking advantage of a large birefringence that can be dynamically controlled by applying external fields, have shown great potential to actively modulate the electromagnetic waves over a broad spectrum [15, 16, 47, 49, 50, 53-60].

Tunable LC metamaterials were first demonstrated in a microwave regime by applying external voltage $[53,59]$. The reorientation of the nematic crystals takes a response time of $300 \mathrm{~ms}$ to obtain a frequency shift of $360 \mathrm{MHz}$ with the voltage changing from $0 \mathrm{~V}$ to $100 \mathrm{~V}$ [59]. Khoo et al. theoretically reported an analysis on tunable metamaterials based on coreshell nanospheres randomly dispersed in LC [47]. Werner et al. presented a reconfigurable metamaterial by sandwiching a nematic LC as a substrate into the conventional negative index metamaterial [50]. Wang et al. numerically investigated the tunability of metamaterials using a rigorous treatment for the LC director reorientation [49]. Compared to the control of microwaves, the manipulation of $\mathrm{THz}$ waves can be realized under a lower applied voltage with faster response speed due to the smaller required thickness of LCs. LC metamaterials with real-time control functions, such as polarization controllers [61], absorbers [62], and spatial light modulators [63] are employed in $\mathrm{THz}$ communications, imaging, sensing, detection, and other fields [64-69]. In 2013, Padilla et al. designed and implemented an LC-based tunable metamaterial absorber in the $\mathrm{THz}$ regime for the first time [70]. Subsequently, they also designed a $\mathrm{THz}$ spatial light modulation which could be independently controlled by a single pixel, and the overall modulation depth of the device reached 75\% [71]. In 2017, Yang et al. used liquid crystals to achieve tunable $\mathrm{THz}$ metamaterial-like electromagnetically induced transparency and electromagnetically induced absorption, with modulation depths of $18.3 \mathrm{~dB}$ and $10.5 \mathrm{~dB}$, respectively [72]. Vasić et al. realized an electrically adjustable liquid crystal $\mathrm{THz}$ metamaterial polarization converter based on a metal dielectric metal cavity structure [61]. Shen and others designed and implemented a multi-functional $\mathrm{THz}$ metamaterial device integrated with liquid crystal, which further expanded the application of liquid crystal metamaterial devices in the $\mathrm{THz}$ regime [73].

In this context, we will give a brief review for the development of tunable metamaterials incorporating liquid crystals. In Section 2, we will discuss the analytical approach for liquid crystals inside metamaterials in detail. Section 3 presents tunable electromagnetic parameter metamaterials based on liquid crystals. A survey of the recent novel liquid crystal-based developments and application is conducted in Section 4. Finally, we will discuss some of the major limitations of LCs and envision a promising future of tunable metamaterials incorporating liquid crystals. Hopefully this review can promote the research on tunable and reconfigurable metamaterials with various active inclusions for the improvement of the operation bandwidth and smart electromagnetic responses.

\section{ANALYTICAL APPROACH FOR LIQUID CRYSTALS INSIDE METAMATERIALS}

During previous analysis for LCs incorporated into an artificial microstructure, an isotropic treatment was found to be effective provided that the EM fields exhibited isotropic field distributions as is the case for most of the $2 \mathrm{D}$ photonic crystals. This means that only consideration of the effective permittivity change of anisotropic LC along the incident electric field axis is required. For metamaterial, such an assumption is more doubtful and clearly the anisotropic distribution has to be taken into account.

In this section, we present a comparison of anisotropic and isotropic treatments for a C-type metamaterial. As shown in Figure 1, the SRR design used in the present work is composed of 

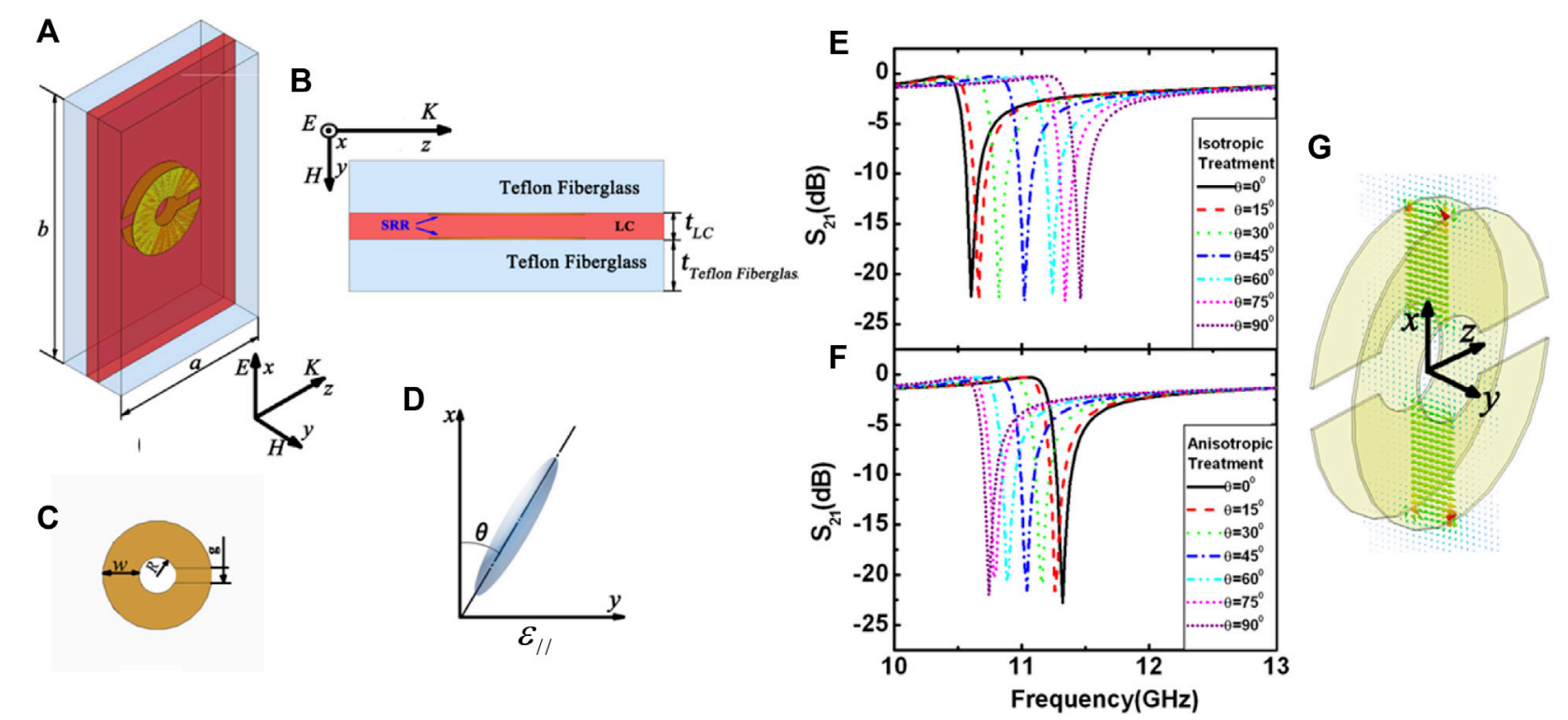

FIGURE 1 | Tunable broadside-coupled SRR metamaterial based on anisotropic LC (A) 3D view (B) top view of the basic unit cell, and (C) front view of SRR (D) Schematic diagram of director. A 5 C B nematic LC is considered in this manuscript $\left(n_{0}=1.50\right.$ and $\left.n_{e}=1.68\right)$. The transmission spectra of the basic unit cell as a function of reorientation angle of $L C$ molecules when the isotropic (E) and anisotropic treatments (F) of $L C$ are employed (G) Localized electric field distribution of SRR in the $x-y$ plane when it is resonated (A)-(G) Reproduced with permission. Copyright 2008, AIP Publishing [54].

double C-shaped strips with a back-to-back direction (Figure 1A) to avoid a magneto-electric response. The metal patterns are printed onto the surface of fiberglass slabs made by Teflon with voids, and then permeated with nematic LC [54]. It is assumed that the electric field of the incident beam is polarized along the $x$ direction and the magnetic field of the incident beam is polarized along the $y$ direction to illuminate the structure along $z$.

The LC layer is treated as a homogenous isotropic material during the first analysis. For aligned nematic liquid crystal, the electric field of the incident beam is polarized along the $x$ direction (Figure 1), and its dielectric constant is approximately given as [56]:

$$
\varepsilon=\frac{\varepsilon_{/ /} \varepsilon_{\perp}}{\varepsilon_{/ /} \sin ^{2} \theta+\varepsilon_{\perp} \cos ^{2} \theta}
$$

Where $\varepsilon_{\perp}$ and $\varepsilon_{/ /}$represent the respective permittivities for the polarized beam perpendicular and parallel to the director axis $\mathrm{n}, \theta$ denotes the rotation angle of the molecular director with respect to the $x$ axis.

On the other hand, a rigorous tensor description for LC is introduced for the anisotropic permittivity of LC. For the LC plate with the initial vector of the molecules in the $x$ direction, the director axis $\mathrm{n}$ can take all values $\{\cos \theta, \sin \theta, 0\}$ by applying a magneto/electric field based on the Fréedericksz effect. LC permittivity is described as follows $[49,55]$ :

$$
\varepsilon=\left(\begin{array}{ccc}
\varepsilon_{\perp}+\Delta \varepsilon \cos ^{2} \theta & 0 & \Delta \varepsilon \cos \theta \sin \theta \\
0 & \varepsilon_{\perp}+\Delta \varepsilon \sin ^{2} \theta & 0 \\
\Delta \varepsilon \cos \theta \sin \theta & 0 & \varepsilon_{\perp}
\end{array}\right)
$$

Where $\Delta \varepsilon=\varepsilon_{/ /}-\varepsilon_{\perp}$
Figure 1 shows the frequency dependence of the metamaterial transport response on the liquid crystal reorientation angle. For the isotropic treatment (Figure 1E), when the LC orients from $0^{\circ}$ to $90^{\circ}$, the resonance frequency of SRR increases gradually from 10.9 to $11.4 \mathrm{GHz}$ whose change is $0.8 \mathrm{GHz}$. On the contrary, for an anisotropic treatment as shown in Figure 1F, the resonance frequency of SRR shifts toward a lower frequency with the increase of the liquid crystal orientation angle. Figure 1G shows the electric field distribution in the $x-y$ plane when the SRR is resonated. It is observed that the localized electric field around SRR is mostly concentrated on the space between two $\mathrm{C}$ strips and parallel to the $y$-direction, instead of being polarized along the $x$-direction where the incident beam is polarized. Therefore, isotropic treatment is no longer so valid for the tunability analysis for the metamaterial [55]. As a matter of fact, the capacitance of the SRR depends on the permittivity component of $\varepsilon_{x}, \varepsilon_{y}$, and $\varepsilon_{z}$, of which $\varepsilon_{y}$ dominates as shown in Figure 1C. When an external field orientates the liquid crystal director from paralleling $\mathrm{x}$ to paralleling $\mathrm{y}, \varepsilon_{y}$ will grow from $\varepsilon_{0}$ to $\varepsilon_{e}$, resulting in an increase in the capacitance and a decrease in the resonance frequency.

\section{TUNABLE ELECTROMAGNETIC PARAMETER METAMATERIALS BASED ON LIQUID CRYSTALS}

\subsection{Tunable Negative Permeability Metamaterial}

In this section, we will introduce two types of negative permeability metamaterial with frequency tunability via LC 

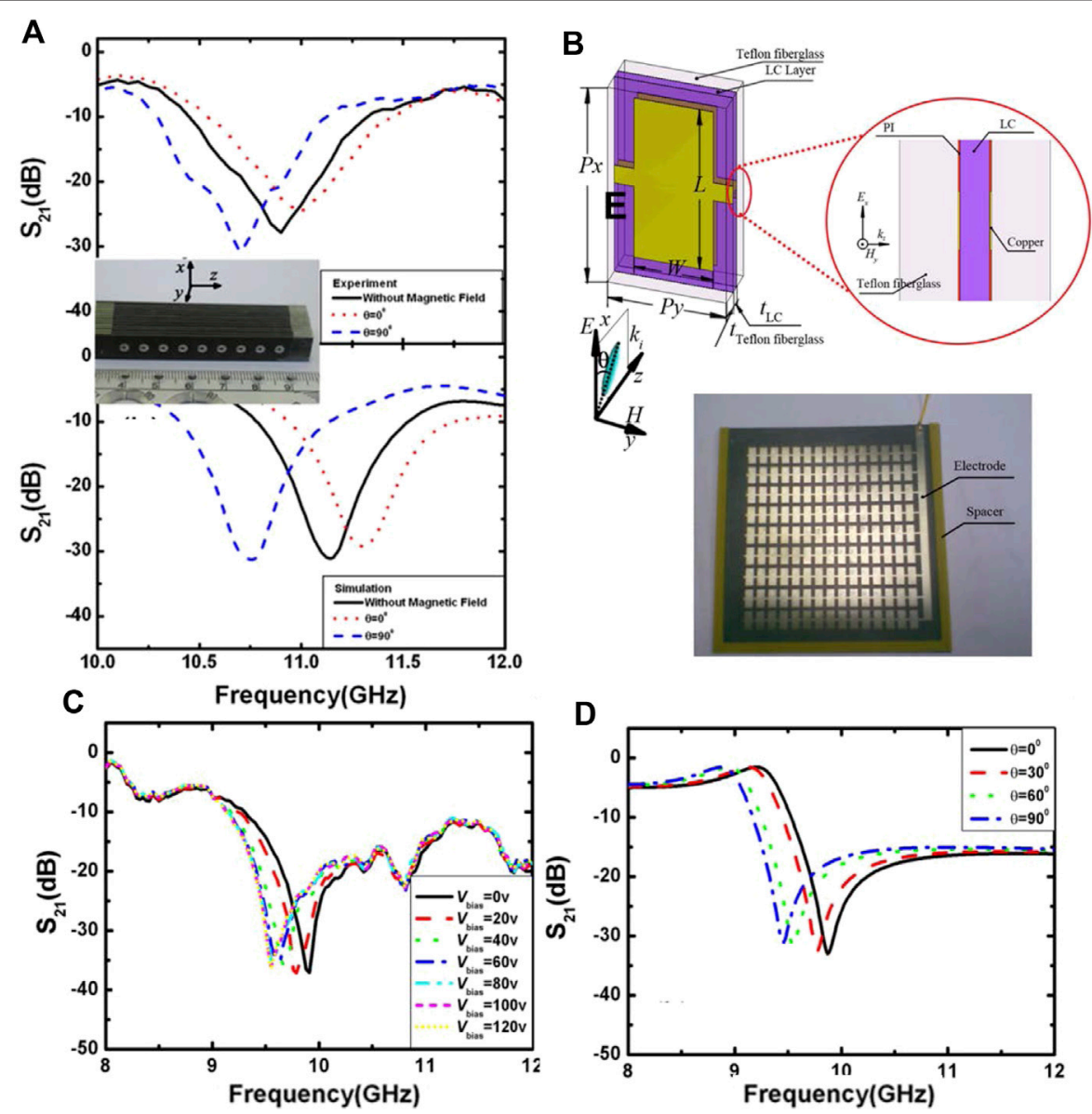

FIGURE 2 | (A) Demonstration of magnetically tunable SRR metamaterial. Inset is the view for the SRR prototype prepared for the infiltration of LC (B) Tunable short-wire pair-type of negative permeability metamaterial based on anisotropic LC (C) Experimental transmission magnitude for short-wire pair metamaterial as a function of external DC bias voltage (D) Numerical prediction of transmission spectra versus LC reorientation angle (A) Reproduced with permission. Copyright 2012, AlP Publishing [54] (B)-(D) Reproduced with permission. Copyright 2008, AIP Publishing [59].

reorientation. In Figure 1, a typical broadside coupling SRR incorporates an LC layer between two reversed C-type metallic patterns. The prototype sample was prepared using printed circuit board technology, as shown in the inset of Figure 2A [54]. The transfer rate of the tunable SRR archetype is measured in the following three conditions; i) the SRR without magnets (in this case the average permittivity is $\varepsilon_{0}=\left(2 \varepsilon_{\perp}+\varepsilon_{/ /}\right) / 3$, ii) applying the magnetic field along $\mathrm{x}(\theta=90)$, and iii) along $\mathrm{y}(\theta=90)$. The calculated results and experimental shown in Figure 2A indicate that a noticeable decrease occurs at $10.9 \mathrm{GHz}$ for the instance without the external magnetic field. The resonance frequency increases to 11.0 and $10.7 \mathrm{GHz}$ when the magnetic field is then applied parallel to the $x$ and $y$ axes, respectively. It can be seen that the dependency of the resonance frequency to the liquid crystal director reorientation corresponds to the calculating results that involve the description of the permittivity tensor of the nematic substance.

Unlike an SRR operating under grazing incidence with magnetic field perpendicular to its surface, Figure 2B shows an electrically tunable short-wire pair-type of negative permeability metamaterial which can work under normal excitation of electromagnetic waves [59]. The unit cell of tunable metamaterial is made up of a couple of short wires put on the surface of fiberglass slabs made from Teflon with voids in between which were infiltrated with a nematic compound. And by adding an extra couple of narrower inplane bars to connect neighbor unit cells. It can be assessed that such interconnecting bars do not change the magnetic resonance behavior of a short-wire pair. To give a preliminary orientation of LC, on the surface of the Teflon substrate and copper element is covered with a thin layer of polyimide (PI) to compel the nematic liquid crystal parallel to the short-wire surface in an alignment state $[57,59]$.

The transmission spectra for the short-wire metamaterial as a bias voltage function are given in Figure 2C. For zero-bias voltage, an obvious decrease takes place at $9.91 \mathrm{GHz}$, around that the effective permeability is anticipated to be negative. And the resonant frequency falls to $9.55 \mathrm{GHz}$ as bias voltage gradually grows from 0 to $100 \mathrm{~V}$. Then the saturation was adjusted and even the bias voltage was increased further. Therefore, the total 


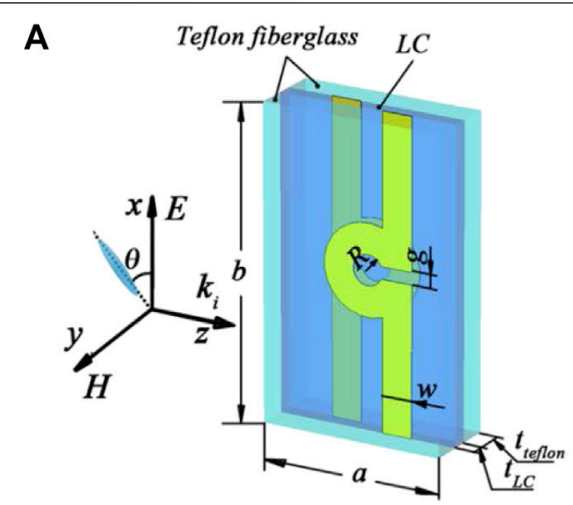

C

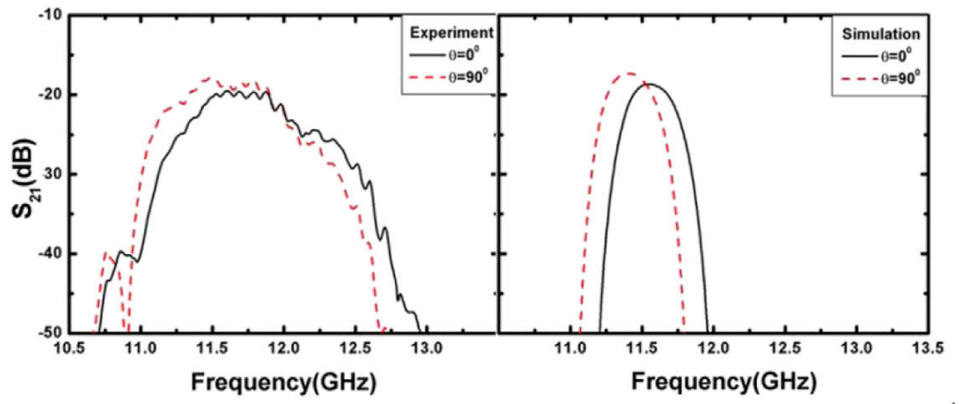

B

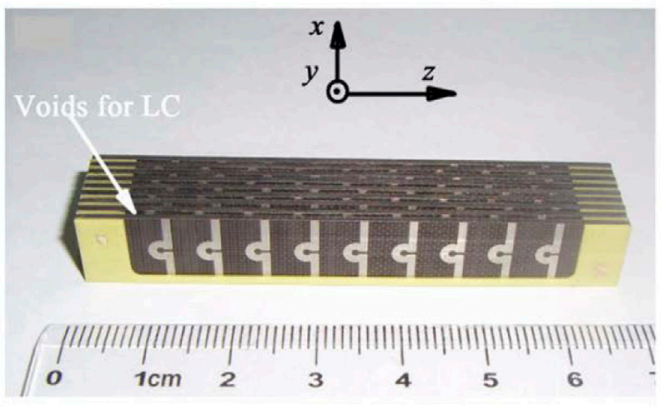

D

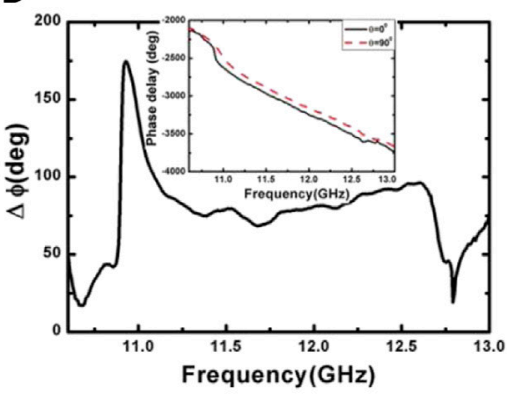

FIGURE 3 | (A) Schematic of the basic unit cell of the tunable negative index metamaterial as well as the reorientation of the LC molecule in the X-y plane (B) Closeup view of the mid-plane of the sample with the other part removed to clarify the configuration of the voids (C) The experimental and simulation transmission responses of the metamaterial with infiltration by LC under different molecules orientations: $\theta=0^{\circ}$ (solid black line) and $90^{\circ}$ (dashed red line), respectively (D) The measured phase shift for the tunable metamaterial when the LC molecules are reorientated from $\theta=0^{\circ}-90^{\circ}$. The inset shows the phase delays for the metamaterial under different reorientations of LC (A)-(D) Reproduced with permission. Copyright 2008, OSA Publishing [58].

frequency shift of $360 \mathrm{MHz}$ can be achieved experimentally. From the full wave numerical calculation for the transmission spectra as a function of the LC molecular reorientation angle, as shown in Figure 2D, it is obvious that when the director of LC is redirected from $0^{\circ}$ to $90^{\circ}$, magnetic resonance frequency of the short-wire pair gradually reduces to $9.45 \mathrm{GHz}$, which results in a $0.42 \mathrm{GHz}$ variation. A slightly narrow frequency variation is most probably due to the existence of a pre-tilted angle of liquid crystal via the PI surface. This means actual LC reorientation will be less than $90^{\circ}$, resulting in less permittivity alteration as well as the changes of resonance frequency.

\subsection{Negative Index Metamaterial}

On the basis of the successful demonstration of a frequency tunable metamaterial with a single negative parameter, we will present a double negative metamaterial with a dynamically varied left-handed passband. The idea is to add negative permittivity via a wire array to the original negative components. In view of tunability requirements, it is necessary to employ unit cells with a combination of negative permittivity and negative permeability parts. Figure 3 shows a tunable omega metamaterial sample with a broadside coupling SRR connected with infinite wire [58]. By utilizing external magnets, it is able to orientate the LC molecular director angle with respect to the $x$ axis from $0^{\circ}$ to $90^{\circ}$.
Figure 3C shows the experimental transmission spectra of the metamaterial. When applying the magnetic field along the $x$ axis $(\theta=0)$, the omega array shows a clear transfer passband, and it has a negative index of refraction as expected, of which the peak is located at $11.72 \mathrm{GHz}$, whereas a redshift of the passband can be observed with the peak shifts at $11.50 \mathrm{GHz}$ under an orthogonal magnetic field excitation $(\theta=90)$. Although this passband deviation of $210 \mathrm{MHz}$ is not wide, it is clearly perceptible with the comparatively broad bandwidth. The frequency shift is in accordance with numerical results calculated using the full tensor description for LC permittivity, as shown in Figure 3C. Experimental broader bandwidth is mainly due to a slight variation of the voids interspacing since the Teflon fiberglass is not so rigid, resulting in different magnetic resonances between layers, causing multi conjoined passbands and consequently a wider transmission window. Figure 3D plots the phase shift for the omega sample with different LC direction angles. From the variation of phase shift, it can be seen that phase difference increases around $10.6 \mathrm{GHz}$ and reaches a peak of $174.5^{\circ}$ at 10.93 $\mathrm{GHz}$, corresponding to an approximate index variation of 0.25 for a 5.4-cm-long sample along the propagation path.

Figure 4 shows the schematic view of the fishnet type of negative index metamaterial [60]. Due to the intrinsic structural characteristic, one layer of unit cells was connected to the other, therefore designing extra connecting bars for bias voltage is not 

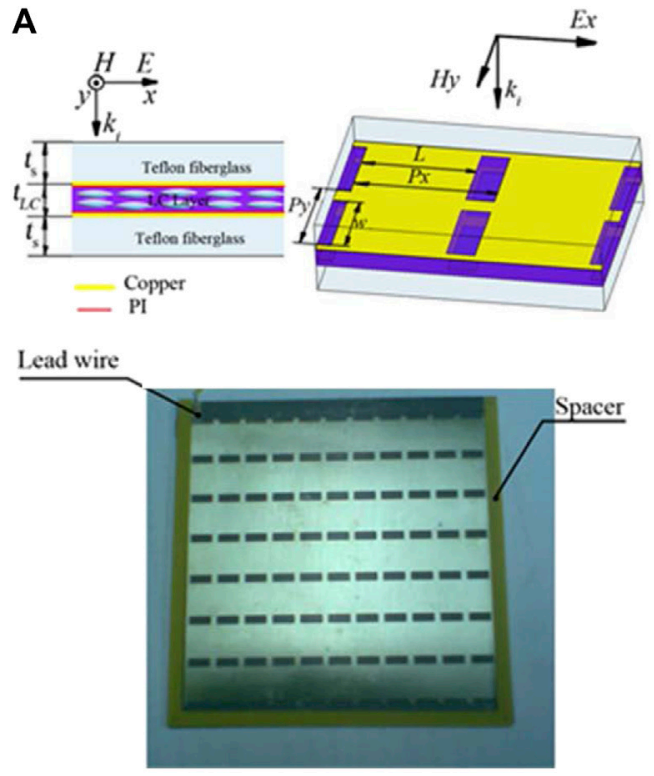

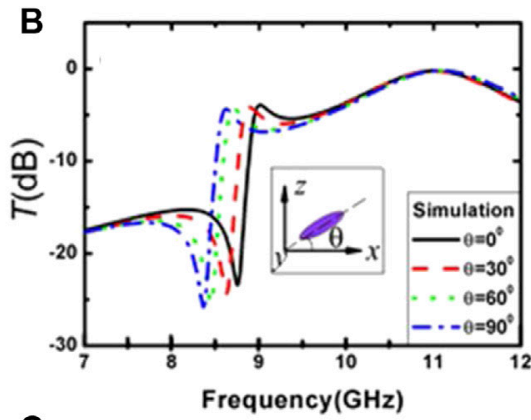

C

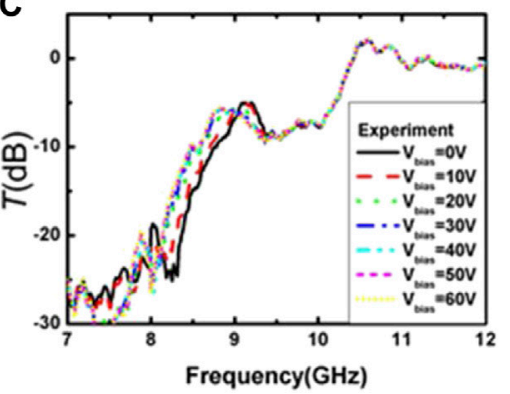

FIGURE 4 | (A) Tunable fishnet type of NIM based on nematic LC. Side view, 3D view of four elementary cells and photograph of metamaterial sample (B) Simulated transmission of tunable fishnet metamaterial when the LC director was reorientated. The inset is the schematic view of LC molecular reorientation in the $x-z$ plane (C) Experimental transmission magnitude of the fishnet metamaterial as a function of external DC bias voltage (A)-(C) Reproduced with permission. Copyright 2008, OSA Publishing [60].

required. Figure 4 gives the transmission spectra variation of the fishnet sample. As shown in Figure $\mathbf{4 B}$, toward the initial target of liquid crystal director parallel to the fishnet surface, there is a well-resolved and high strength transfer summit around $9.01 \mathrm{GHz}$. The liquid crystal director redirecting from $0^{\circ}$ to $90^{\circ}$ shows that the ground transfer summit moves downward to $8.60 \mathrm{GHz}$. Figure 4C demonstrates the fishnet array's measured transmission spectra under different bias voltages. For zero-bias voltage, the fishnet structure displays a transfer passband around $9.14 \mathrm{GHz}$ and another higher passband starting at $10.60 \mathrm{GHz}$ with a smaller decrease in the gap. Quantitatively, as the bias voltage grows up to $60 \mathrm{~V}$, the first transfer summit moves from $9.14 \mathrm{GHz}$ downward to $8.80 \mathrm{GHz}$, accounting for a frequency shift of $340 \mathrm{MHz}$. The frequency shift for the passband is saturated beyond $60 \mathrm{~V}$, indicating that the LC director is nearly perpendicular to the surface of the fishnet pattern.

\section{RECENT DEVELOPMENT AND APPLICATIONS}

\subsection{Tunable Metamaterials With Liquid Crystals}

Liquid crystals have the dual properties of crystals and fiuids. As mentioned in Section 3, LC reorientations would result in a dramatic change in the refractive index of LC, which in turn, would further change the response to electromagnetic waves. In nematic LCs, the crystal is in a rod-shape and the external stimulations such as thermal heating, pressure, and the magnetic and electric field, can control its directional order, resulting in good refractive index tunability [42, 59, 74-91]. Therefore, LCs are extensive used as the liquid background of solid metamaterials $[51,52,92]$. The manipulations on the LC background metamaterial are first protested against in the microwave regime. The resonant frequency of the metamaterial is shifted under a DC voltage [93-97]. Compared to the operation under microwave conditions, the control of the $\mathrm{THz}$ wave can be obtained with a much faster response at a lower electrical voltage since the needed thickness of LC is much smaller [69, 98, 99]. For liquid crystals in a particular phase such as NLC, it is necessary to discuss their collective crystal responses. The timescale of collective crystal response is usually in the order of milliseconds-microseconds. The thickness of the liquid crystal will influence its response speed. To achieve certain modulations requires a very thick LC layer, leading to several disadvantages such as high operating voltage, slow response, and poor pre-alignment. A large birefringent liquid crystal material can reduce the thickness of the liquid crystal device, ensure the good orientation of the liquid crystal, and improve the response speed of the device. For a liquid crystal layer of the same thickness, it can increase the modulation range of the liquid crystal device. Compared with the thermal and UV irradiation-induced tunability of optical metamaterials, which have been experimentally tested, showing a slow temporal response, electrical tuning has a faster time response, which is the most attractive due to its easy integration and high reliability. 

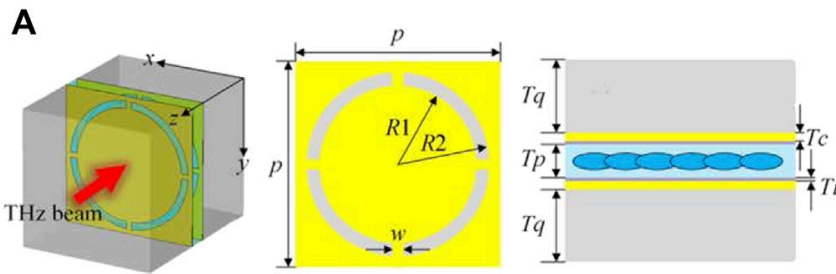

B

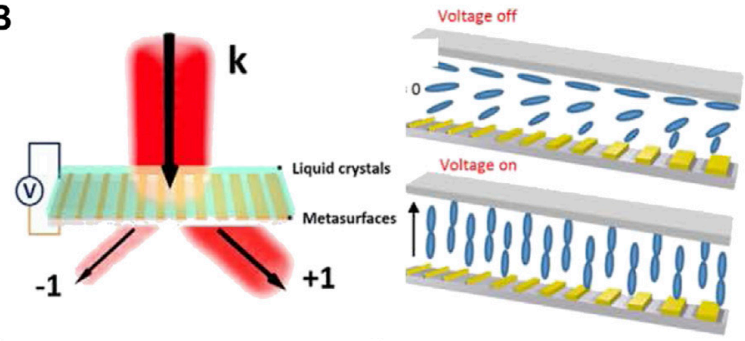

C THz waves

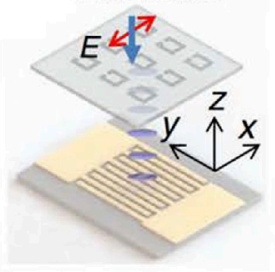

$0 \mathrm{~V}$

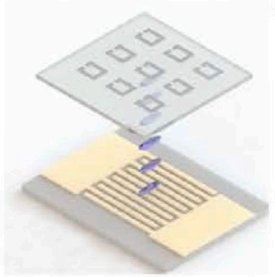

$80 \mathrm{~V}$ at $1 \mathrm{kHz}$

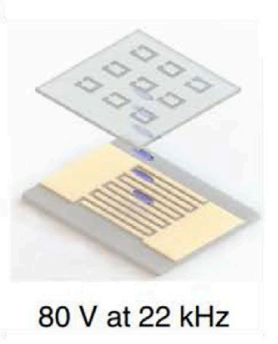

D

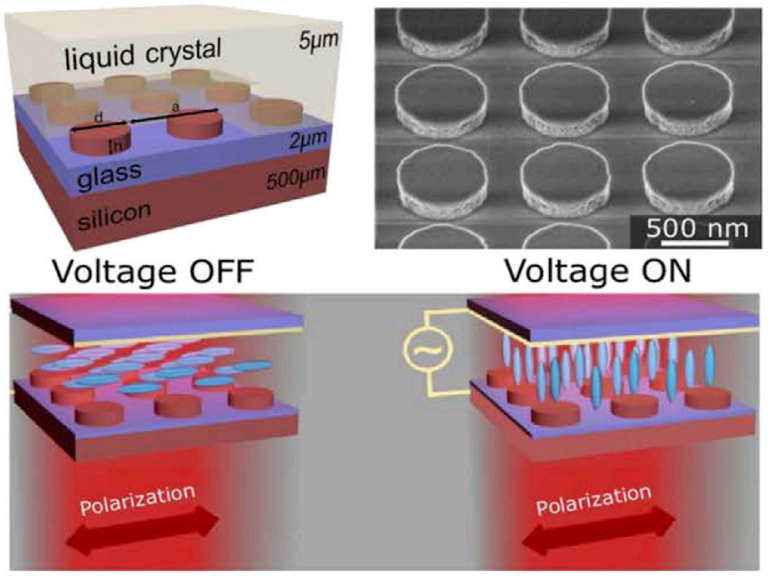

E

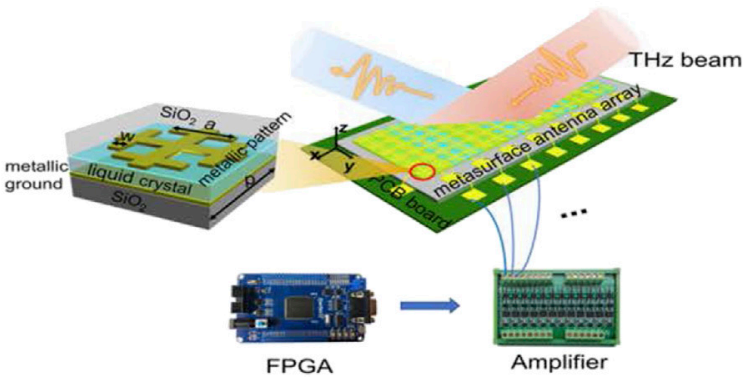

FIGURE 5 | (A) 3D schematic diagram of a unit cell, top view of the metal resonance unit cell and side view of the unit cell (B) Schematic of a reconfigurable binary grating meta-surface as part of a LC cell and the binary gratings combined with nematic LC when the voltage is on and off (C) Experimental spectra of the IPS DFLC cell with the metamaterial, schematic drawing of DFLC director orientations and simulated spectra of the cell at applied voltages of $0 \mathrm{~V}, 80 \mathrm{~V}$ at $1 \mathrm{kHz}$, and $80 \mathrm{~V}$ at $22 \mathrm{kHz}$ (D) Sketch of a silicon nanodisk meta-surface integrated into the LC cell. SEM image of a fabricated silicon nanodisk meta-surface. Schematic of the LC alignment for no applied voltage ("off" case) and for the case when a moderate voltage is applied between the two electrodes of the LC cell ("on" case). The red arrow indicates the polarization of the incident light (E) The schematic of the THz programmable meta-surface. The unit cell consisting of an LC layer embedded into two metallic layers is shown in the left inset (A) Reproduced with permission. Copyright 2019, OSA Publishing [82] (B) Reproduced with permission. Copyright 2016, OSA Publishing [91] (C) Reproduced with permission. Copyright 2015, OSA Publishing [79] (D) Reproduced with permission. Copyright 2017, AIP Publishing [81] (E) Reproduced with permission. Copyright 2020, AIP Publishing [69].

Recently, a nematic liquid crystal-based tunable terahertz metamaterial which has large modulation depth and low insertion loss was designed [82]. The structure of the proposed compositive metamaterial is shown in Figure 5A, which consists of a two-layer sub-wavelength circular air loop array fabricated on a quartz glass substrate and embedded into the liquid crystal. The obtained results show that the amplitude modulation depth and insertion loss for normally incident electromagnetic waves are about $96 \%$ and $1.19 \mathrm{~dB}$ at $0.4212 \mathrm{THz}$, respectively, with the bias voltage varying from 0 to $16 \mathrm{~V}$. Meanwhile, the transmission peak frequency gradually decreases from 0.42 to $0.38 \mathrm{THz}$. Differing from the resonant frequency shifted under a DC voltage, another literature paper about the continuously tunable and fast-response terahertz metamaterial was studied using in-plane switching (IPS) dual-frequency liquid crystal (DFLC) cells, in which dielectric anisotropy could be changed with the variation of applied voltage frequency [79]. When the frequency of an external voltage is switched, the resonance peak of the metamaterial can move to the high frequency or the low frequency, and the response times of the redshift and blueshift are 1.044 and $1.376 \mathrm{~ms}$, respectively. Figure 5C shows the dual- frequency liquid crystal director orientations at the three applied voltages (voltages of $0,80 \mathrm{~V}$ at $1 \mathrm{kHz}$, and $80 \mathrm{~V}$ at $22 \mathrm{kHz}$ ). More recently, a $\mathrm{THz}$ programmable meta-surface integrated with LC was proposed to manipulate the $\mathrm{THz}$ beam dynamically [69]. Figure $\mathbf{5 E}$ shows the $3 \mathrm{D}$ view of the $\mathrm{THz}$ programmable meta-surface. In Figure 5E, a 24-element linear array consists in the meta-surface, and a multichannel amplifier is in connection with each element. The control signal is outputted by the FPGA, and the amplified signals are utilized to control the phase of every element individually.

By further shrinking LC background metamaterials, the realtime control of IR and visible light are realized. Andrei Komar et al. presented an electrically tunable all-dielectric optical metasurface consisting of silicon nanodisks embedded into liquid crystals, as shown at Figure 5D [81]. The reorientation of nematic liquid crystals under an external electric field can change the anisotropic permittivity tensor of the meta-surface, which in turn, alters the response to electromagnetic waves. By controlling voltage "on" and "off" to achieve reorientation of the nematic liquid crystals and obtain $75 \%$ modulation depth and a phase change of up to approximately $\pi$. Similarly, a new 


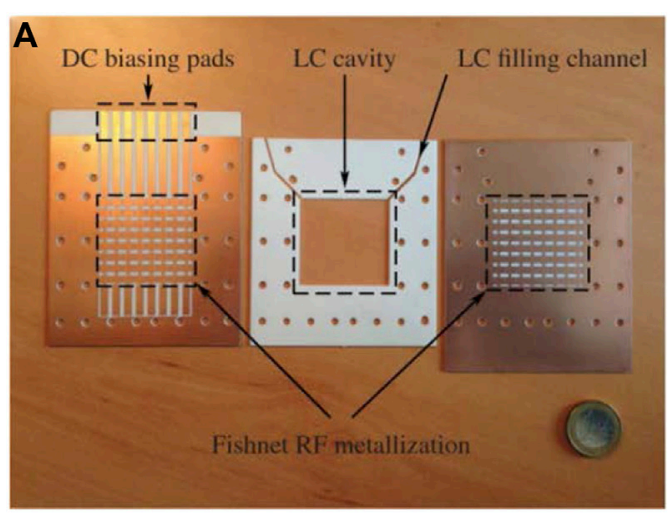

B
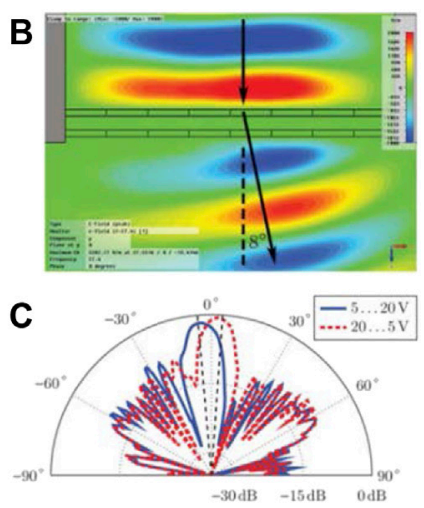

D

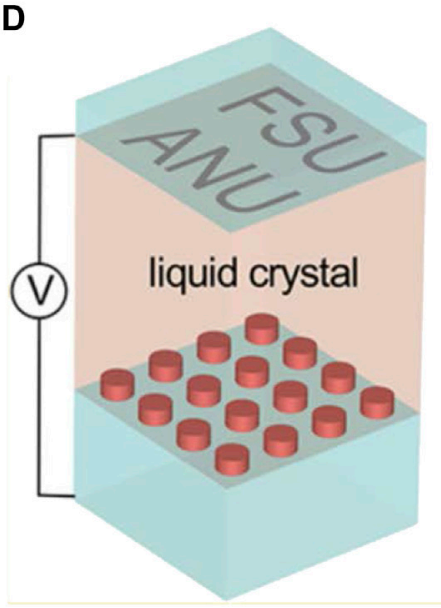

E
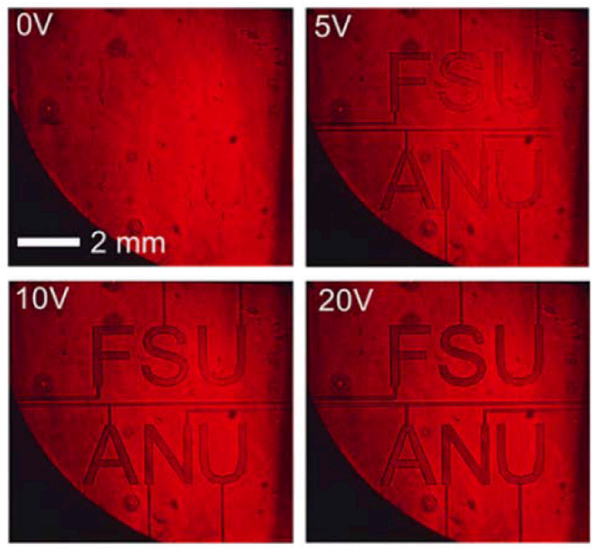

$20 \mathrm{~V}$

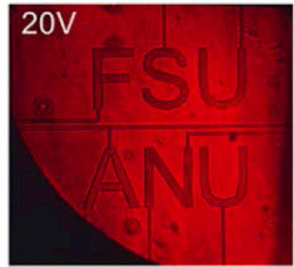

FIGURE 6 | (A) Photograph of a single opened fishnet unit-cell layer (B) Simulated electric near-field distribution of the two-layer fishnet array with a gradient of the LC permittivity at $27.4 \mathrm{GHz}$ (C) Measured normalized far-field patterns at $27.5 \mathrm{GHz}$ for two tuning states (D) Electrical tuning of the LC meta-surface (E) Real color images of the meta-surface at 0, 5, 10, and $20 \mathrm{~V}$ recorded in transmission (A)-(C) Reproduced with permission. Copyright 2014, IEEE [78] (D)-(E) Reproduced with permission. Copyright 2019, American Chemical Society [83].

combination of nematic liquid crystals and a binary-grating meta-surface was presented to control the diffraction efficiency by altering the applied voltage in the visible region, as shown in Figure 5B [91].

In addition, the effective tuning of the LC background metamaterial for wavefront manipulation has a wide prospect such as in tunable gradient indexed lenses [78, 94]. By introducing a voltage gradient over the fishnet metamaterial based on liquid crystals, the phase distribution over the aperture can be tuned, and thus the radiation direction can be manipulated. Thus, we can develop novel applications for wavefront manipulation based on liquid crystal metamaterials. In a recent study based on fishnet metamaterials, the unit cells of the fishnet were embedded with nematic liquid crystal which provided a continuous tuning of each column of the array [78]. The photograph of a single opened fishnet unit-cell layer is shown in Figure 6A. Figure 6B shows the simulated electric field distribution at $27.4 \mathrm{GHz}$. By using liquid crystal as a tunable dielectric layer, the authors could achieve a voltage-tunable phase gradient over the aperture array, and obtain a measured continuous maximum beamscanning angle of $5^{\circ}$ at $27.5 \mathrm{GHz}$, as shown in Figure $6 \mathrm{C}$. The meta- surface based on liquid crystals can also be used to achieve tunable transparent displays for visible light [83]. As shown at Figure 6D, an all-dielectric meta-surface composed of silicon nanocylinders integrated into a nematic liquid crystal cell is presented. A switchable dielectric meta-surface display is achieved by varying the applied voltage from $0 \mathrm{~V}$ to $20 \mathrm{~V}$ in steps of $5 \mathrm{~V}$. Figure $6 \mathrm{E}$ shows the real-color images of the proposed meta-surface at $0,5,10$, and $20 \mathrm{~V}$ recorded in transmission. It is worth noting that the demonstrated meta-surface display uses a different operation theory than normal liquid crystal displays.

In addition to the applied voltage, temperature can be also used as an external control parameter [57, 76, 85, 100-105]. Heating of the sample over the liquid crystal transition temperature leads to the phase transition of the liquid crystal between nematic and isotropic, which can be used to realize the tunability of response to electromagnetic waves. In 2009, a thermally tunable optical metamaterial was demonstrated based on aligned nematic liquid crystals in the visible range [57]. Structure of the coupled sample is shown in Figure 7A. By changing the ambient temperature (from $20^{\circ}$ to $50^{\circ} \mathrm{C}$, which is beyond the phase transition temperature $\mathrm{T}_{\mathrm{c}}, 35^{\circ} \mathrm{C}$ ), the phase 
A

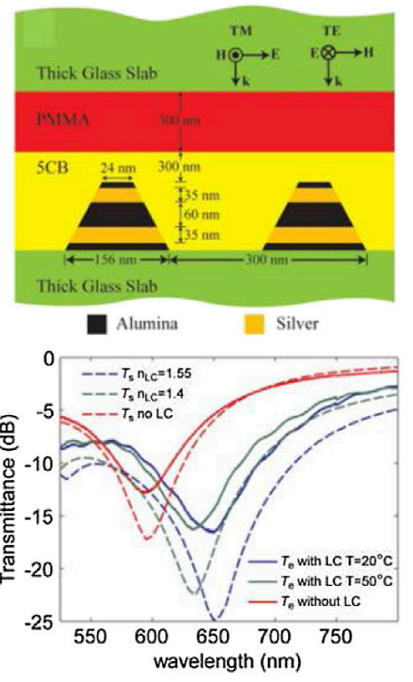

D

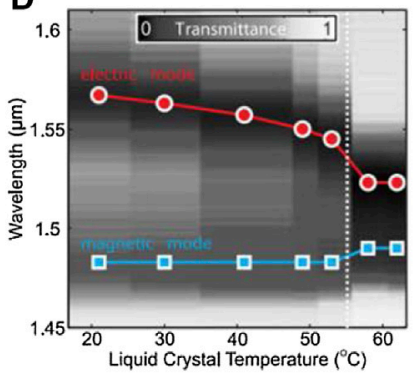

B
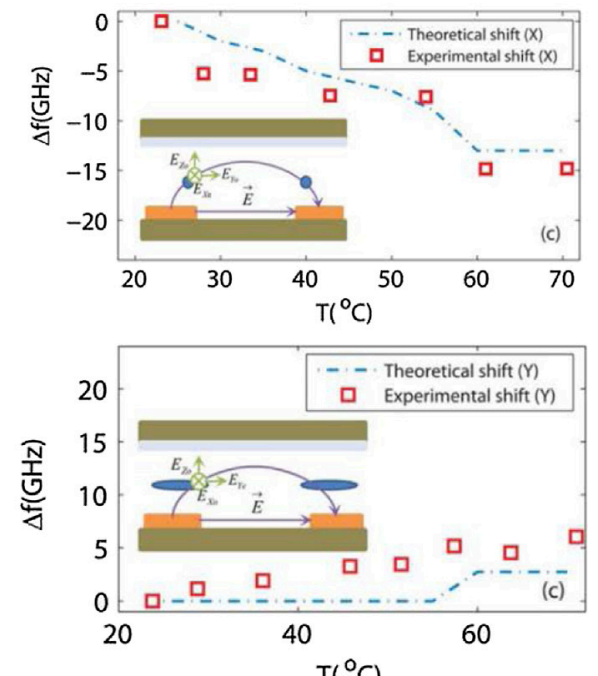

$E^{T}\left({ }^{\circ} \mathrm{C}\right)$
C

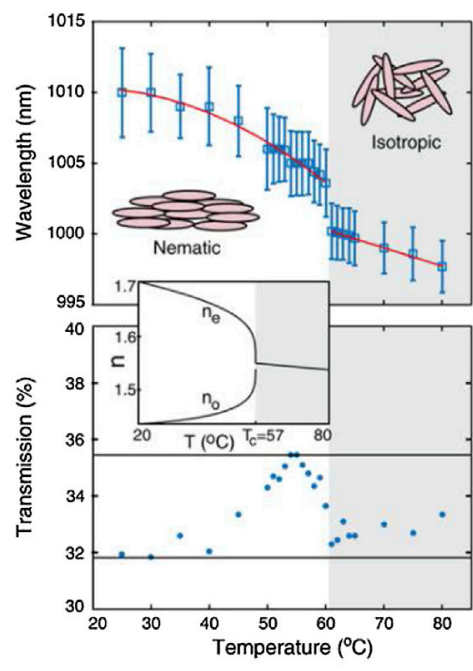

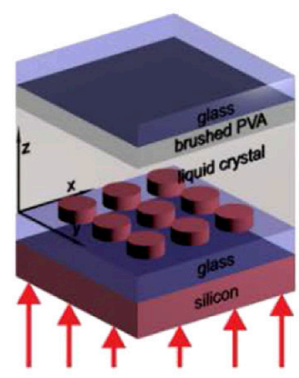

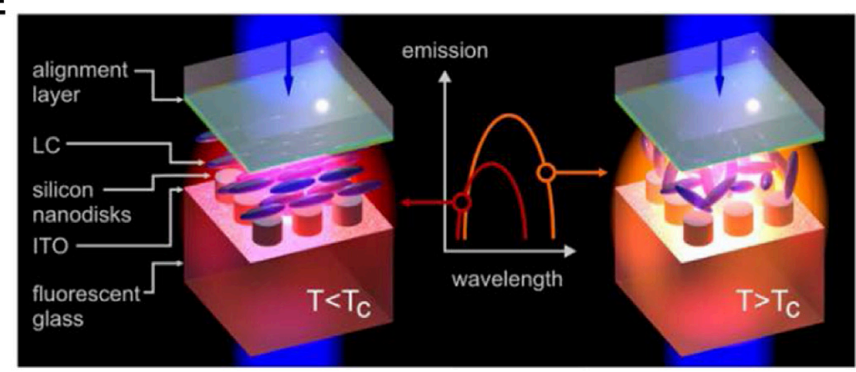

FIGURE 7 | (A) Structure of the coupled nanostrip sample and demonstration of a thermally tunable magnetic response in a metamaterial. (B) Experimentally measured results with the LC aligned in the $x$ - and $y$-direction. (C) The shift of the resonance with temperature and the minimum transmission of the resonance as a function of temperature. The inset shows the temperature dependence of the refractive index. (D) Schematic of the silicon nanodisk meta-surface integrated into an LC cell and the temperature-dependent transmittance spectra of electric/magnetic modes. (E) Sketch of a silicon nanocylinder meta-surface integrated into a liquid crystal cell. When the liquid crystal is heated, it changes its state from nematic to isotropic, resulting in a spectral shift of the meta-surface resonances and the tuning of the emission enhancement. (A) Reproduced with permission. Copyright 2009, AIP Publishing [57]. (B) Reproduced with permission. Copyright 2013, IEEE [76]. (C) Reproduced with permission. Copyright 2017, AIP Publishing [102]. (D) Reproduced with permission. Copyright 2015, American Chemical Society [100]. (E) Reproduced with permission. Copyright 2018, American Chemical Society [103].

transition of nematic LCs from the ordered phase to the isotropic phase can be realized, which in turn means the magnetic response wavelength can be effectively changed, as shown in Figure 7A. Temperature control of metamaterials with liquid crystals can also work in the terahertz region. The tunability of resonant response is realized due to the orientations of liquid crystals by changing temperature [76]. The two arrangements of liquid crystals lead to different modulations to the terahertz wave. It is illustrated that the resonant frequency decreases when the liquid crystal is aligned in the $x$-direction, whereas it increases when the liquid crystal is aligned in the $y$-direction in Figure $7 \mathbf{B}$. In essence, the temperature control of metamaterials with liquid crystal is due to the changes in the refractive index of the liquid crystal. In Figure 7C, the results showed that the shift of the resonance fits the form of the temperature dependence of the liquid crystals' refractive index [102]. Furthermore, dynamic manipulation of electric and magnetic resonances can be both realized based on the temperature-dependent refractive-index tunability of the liquid crystal [100]. As shown in Figure 7D, the very different degrees of tunability of the two resonances modes allows for dynamically adjusting the spectral mode separation. In this experiment, the effect of temperature change on the refractive index of liquid crystal and the influence of phase transition on the resonant mode are both investigated. Besides the realization of dynamic tuning of the electromagnetic response of such metamaterials based on liquid crystals and the dynamic control of the light-emission properties of active liquid crystal meta-surfaces by an external control parameter were also demonstrated by Justus Bohn and coworkers [103]. Figure 7E shows the schematic of a metasurface integrated into a liquid crystal cell. When heating the liquid crystal, its state changes from nematic to isotropic, resulting in a spectral shift of the meta-surface resonances and the enhancement of tuning of the emission.

\subsection{Liquid Crystals-Based Applications}

Liquid crystals have dielectric and optical anisotropy. The directors of liquid crystal molecules can be adjusted through 

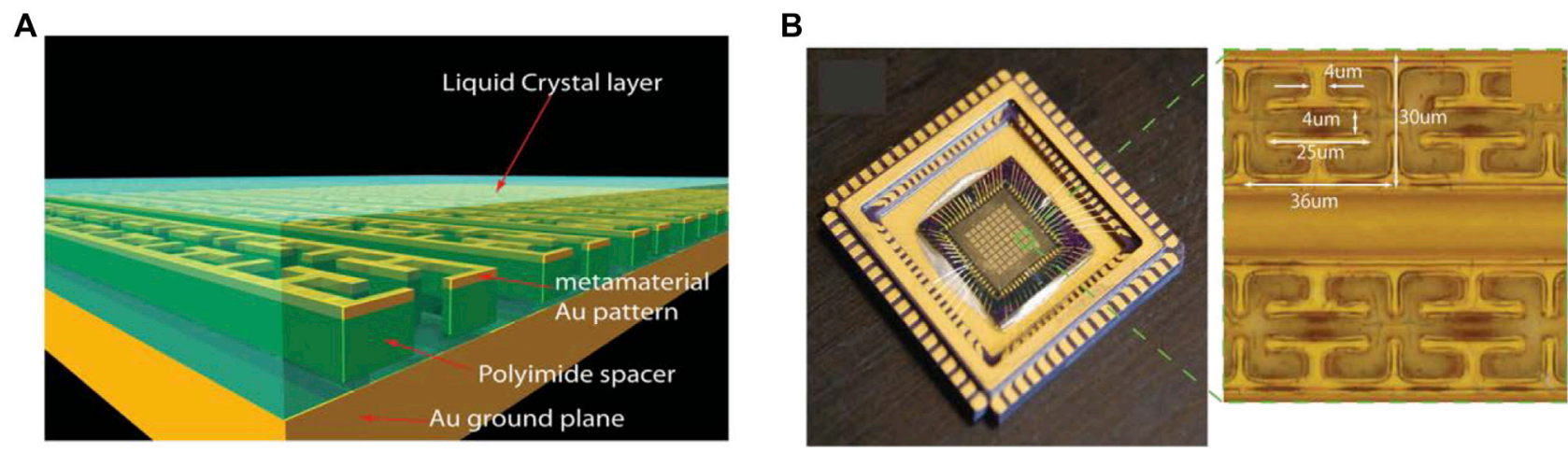

C (d)

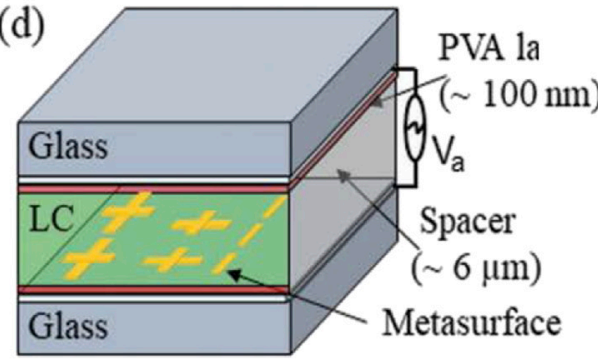

(e)

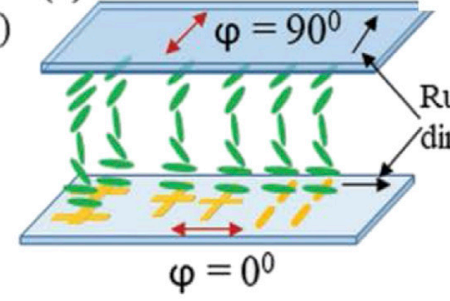

(f)

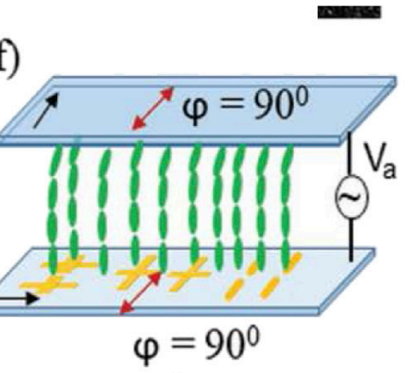

D

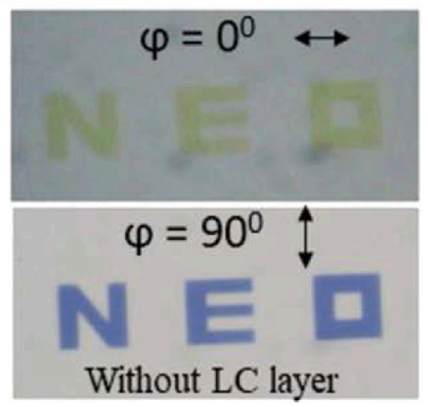

E With LC layer
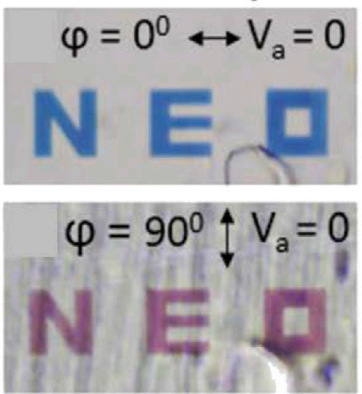
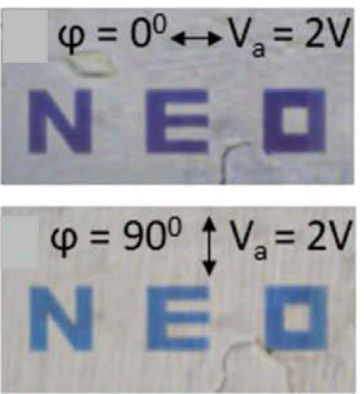
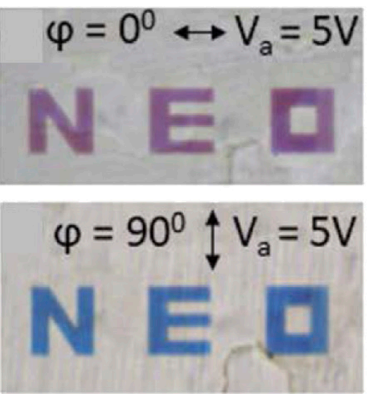

FIGURE 8 | (A) Schematic of the liquid crystal metamaterial absorber spatial light modulator. (B) Camera image of the MMA spatial light modulator device coated with LC. Close-up of MMA unit cells and corresponding dimensions. (C) Schematic view of the integrated liquid crystal meta-surface device and the working principle to rotate the polarization of incident $y$-polarized light. (D) Transmission images of the NEO tag, without an LC layer for $x$-polarized incident light ( $\phi=0^{\circ}$ ) and for $y$-polarized incident light $\left(\phi=90^{\circ}\right)$. (E) Transmission image of the NEO tag, with an LC layer for $\phi=0^{\circ}$ and $\phi=90^{\circ}$ at 0, 2, and 5 V. (A) Reproduced with permission. Copyright 2014, Wiley-VCH [110]. (A), (B) Reproduced with permission. Copyright 2017, American Chemical Society [108]. (C)-(E) Reproduced with permission. Copyright 2020, Wiley-VCH [113].

external fields, thereby effectively controlling the intensity, phase, and polarization of light waves and electromagnetic waves in various frequency bands, giving the liquid crystal materials a huge application potential. Using the controllability of liquid crystals and the special properties of metamaterials, meta-devices with real-time control functions can be realized, such as polarization controllers, absorbers, beam scanning, and spatial light modulators for information communication, imaging, and transmission. It can effectively solve the problems of the slow response speed of traditional liquid crystal devices, and has the advantages of small size, light weight, and easy integration.

The free control of liquid crystal orientation can promote the development of manipulability of metamaterial-based devices. Based on the tunability of the refractive index of liquid crystal, more latent applications of liquid crystal background metamaterials have been discussed such as the perfect absorber [62, 70, 106, 107], polarization converter [108, 109], and modulators [110-113]. Salvatore Savo et al. have illustrated the ability of functional liquid crystals in a metamaterial absorber array working as a spatial light modulator during $\mathrm{THz}$ wavelengths by experiments. The $3 \mathrm{D}$ artistic impression of the metamaterial array is shown in Figures $\mathbf{8 A}$ and $\mathbf{8 B}$ [110]. The narrow band frequency response of the metamaterials can be electronically controlled by the orientation of the liquid crystal molecules. Polarization converters using liquid crystal metamaterials are also demonstrated. The physical underlying principle of efficient polarization converters consists in their operation in the regime in which the radiative losses are larger than the non-radiative ones [108]. The polarization of the incident electric field is linear. Depending on the applied 
voltage, the linearly polarized incident light is converted to RCP, linear, and LCP light. Moreover, in a recent literature paper, an electrically switchable color tag on account of an active liquidcrystal meta-surface platform was realized [113]. The realization of the proposed active color tags utilized the ability of the tunability of nematic liquid crystal reorientations controlled by an external electric field which would rotate the polarization of incident light. Figure $\mathbf{8 C}$ shows the schematic view of the integrated liquid crystal meta-surface device and the working principle to rotate the polarization of incident y-polarized light. Figure 8D shows the transmission pictures of a NEO tag, without an LC layer for x-polarized incident light $\left(\phi=0^{\circ}\right)$ and for y-polarized incident light $\left(\phi=90^{\circ}\right)$. Correspondingly, Figure $8 \mathrm{E}$ shows the transmission image of the NEO tag, with an LC layer for $\phi=0^{\circ}$ and $\phi=90^{\circ}$ at 0,2 , and $5 \mathrm{~V}$.

\section{PROSPECTIVE AND CONCLUSION}

Incorporating active inclusions into metamaterial structures is of fundamental importance for increasing the operation bandwidth and dynamically manipulating electromagnetic waves. Artificially micro/nano-structured metamaterials with nematic LC provide a novel way to actively modulate the electromagnetic response. Nematic liquid crystals (NLC) dominate strong optical nonlinearities for photonics applications covering the femtoseconds to milliseconds time scales, and across a wide spectral range. The combination of the optical nonlinear behavior of liquid crystals and the peculiar physical properties of metamaterials is also one of the important advances in the field of liquid crystals. Many promising results on the tunability of metamaterials incorporated with nematic liquid crystal are reviewed, and these experimental studies have also reflected the inherent drawbacks of nematic liquid crystals as the active component. The foremost is the immobile layer in the immediate vicinity of the plasmonic structures [51]. This together with local inhomogeneous director axis alignment on the nanostructures all act to diminish the effective birefringence of the NLC for modulation/tuning effects. The theoretical tuning range due to complete realignment of the director axis with orthogonally polarized illuminating light is usually larger than the experimentally measured tuning range. This defect can be taken into account in the design of the metamaterial, and the optimization of the microstructure of the metamaterial may promote the agreement between the experimental value and the theoretical value. It is also very urgent and important to further design and fabricate liquid crystal materials with broadband, greater birefringence, smaller loss, faster response speed, and taking into account the wide liquid crystal phase temperature and other excellent characteristics. Furthermore, recent studies of another phase of chiral nematic liquid crystals, namely, blue-phase liquid crystals (BPLC) have presented promising alternatives. The second question that should be discussed is molecular anchoring. The realization of the LC tunability of metamaterials in the near infrared and optical regime is a much harder task, since the effects of molecular anchoring to the nanostructured surfaces becomes important when the dimensions of the individual meta-atoms become comparable to the size of the LC molecules, which has a significant impact on the tunability of optical metamaterials $[114,115]$. For example, under hard-anchoring conditions, the LC director axis in the immediate vicinity of the alignment layer remains unchanged while the bulk undergoes reorientation by the applied field. Liquid-crystal tuning of plasmonic resonances crucially rely on the ability to reorient the LC molecules within the near-fields of the plasmonic structure. One promising way of influencing the anchoring energy of the LC and to enhance tunability is the chemical functionalization of the metamaterial surface. In a recent study, it was found that after reducing the contribution of the supporting substrate to the metamaterial surface area it became possible to minimize the anchoring forces in the resulting hybrid and engage in-plane switching of the LC director at a nanoscale dimension [89].

In this paper, we present a brief review for tunable single/ double negative metamaterials based on nematic LC. And we discuss the development of the tunable behavior of metamaterials induced by liquid crystals. By utilizing an LC director controlled by applied voltage and temperature, it is possible to reversely modulate the metamaterials' operation frequency along with the unique value of effective electromagnetic parameters. LC reorientations will result in a change of the $\mathrm{LC}$ refractive index, which in turn, changes the response to electromagnetic waves. The ease of incorporation and large birefringence of LC enable it to become an ideal candidate for metamaterial substrates with varied permittivity. It is worth noting that the birefringence of LC increases with frequency. It is demonstrated that LC-based metamaterials are promising for the remarkable improvement of the bandwidth and may facilitate related applications at terahertz or even optical regimes. Hopefully this review can promote research on tunable and reconfigurable metamaterials with various active inclusions for the improvement of the operation bandwidth and smart electromagnetic responses.

\section{AUTHOR CONTRIBUTIONS}

FZ and YF conceived the idea. FZ, XJ, and YF prepared the draft. All authors contributed to the discussion and revision of the manuscript.

\section{FUNDING}

The authors would like to acknowledge financial support from National Natural Science Foundation of China (NSFC) (Grants Nos. 61771402, 11674266, 12074314), NPU AoXiang New Star program, Science, Technology and Innovation Commission of Shenzhen Municipality (JCYJ20170817162221169), Shaanxi Province Postdoctoral Science Foundation (No. 2018BSHEDZZ64), and Natural Science Basic Research Plan in Shaanxi Province of China (Nos. 2018JM6024, 2020JM-145). 


\section{REFERENCES}

1. Veselago VG, The electrodynamics of substances with simultaneously negative values of $\varepsilon$ and $\mu$. Sov Phys Usp (1968) 10:509-14. doi:10.1070/ PU1968v010n04ABEH003699

2. Ran L, Huangfu J, Chen H, Li Y, Zhang X, Chen K, et al. Microwave solid-state left-handed material with a broad bandwidth and an ultralow loss. Phys Rev B (2004) 70:073102. doi:10.1103/PhysRevB.70.073102

3. Ran L, Huangfu J, Chen H, Zhang X, Cheng K, Grzegorczyk TM, et al. Experimental study on several left-handed metamaterials. Electromag Waves (2005) 51:249-79. doi:10.2528/pier04040502

4. Dolling G, Enkrich C, Wegener M, Soukoulis CM, Linden S, Simultaneous negative phase and group velocity of light in a metamaterial. Science (2006) 312: 892-4. doi:10.1126/science.1126021

5. Zhao H, Kang L, Zhou J, Zhao Q, Li L, Peng L, et al. Experimental demonstration of tunable negative phase velocity and negative refraction in a ferromagnetic/ferroelectric composite metamaterial. Appl Phys Lett (2008) 93: 201106. doi:10.1063/1.3033397

6. Kang L, Zhao Q, Zhao H, Zhou J, Ferrite-based magnetically tunable lefthanded metamaterial composed of SRRs and wires. Opt Express (2008) 16: 17269-75. doi:10.1364/oe.16.017269

7. Pendry JB, Holden AJ, Stewart WJ, Youngs I, Extremely low frequency plasmons in metallic mesostructures. Phys Rev Lett (1996) 76:4773-6. doi:10. 1103/PhysRevLett.76.4773

8. Pendry JB, Holden AJ, Robbins DJ, Stewart WJ, Magnetism from conductors and enhanced nonlinear phenomena. IEEE Trans Microw Theory Tech (1999) 47:2075-84. doi:10.1109/22.798002

9. Smith DR, Kroll N, Negative refractive index in left-handed materials. Phys Rev Lett (2000) 85:2933-6. doi:10.1103/PhysRevLett.85.2933

10. Shelby RA, Smith DR, Schultz S, Experimental verification of a negative index of refraction. Science (2001) 292:77-9. doi:10.1126/science.1058847

11. Greegor R, Parazzoli C, Li K, Koltenbah B, Tanielian M, Experimental determination and numerical simulation of the properties of negative index of refraction materials. Opt Express (2003) 11:688-95. doi:10.1364/oe.11. 000688

12. Parazzoli CG, Greegor RB, Li K, Koltenbah BE, Tanielian M, Experimental verification and simulation of negative index of refraction using Snell's law. Phys Rev Lett (2003) 90:107401. doi:10.1103/PhysRevLett.90.107401

13. Huangfu JT, Ran LX, Chen HS, Zhang XM, Chen KS, Grzegorczyk TM, et al. Experimental confirmation of negative refractive index of a metamaterial composed of Omega-like metallic patterns. Appl Phys Lett (2004) 84:1537-9. doi:10.1063/1.1655673

14. Chen H, Ran L, Huangfu J, Zhang X, Chen K, Grzegorczyk TM, et al. Lefthanded materials composed of only S-shaped resonators. Phys Rev E Stat Nonlin Soft Matter Phys (2004) 70:057605. doi:10.1103/PhysRevE.70.057605

15. Minovich A, Neshev DN, Powell DA, Shadrivov IV, Kivshar YS, Tunable fishnet metamaterials infiltrated by liquid crystals. Appl Phys Lett (2010) 96: 193103. doi: $10.1063 / 1.3427429$

16. Minovich A, Farnell J, Neshev DN, McKerracher I, Karouta F, Tian J, et al. Liquid crystal based nonlinear fishnet metamaterials. Appl Phys Lett (2012) 100:121113.: doi:10.1063/1.3695165

17. Pendry JB, Negative refraction makes a perfect lens. Phys Rev Lett (2000) 85: 3966-9. doi:10.1103/PhysRevLett.85.3966

18. Fang N, Lee H, Sun C, Zhang X, Sub-diffraction-limited optical imaging with a silver superlens. Science (2005) 308:534. doi:10.1126/science.1108759

19. Liu Z, Durant S, Lee H, Pikus Y, Fang N, Xiong Y, et al. Far-field optical superlens. Nano Lett (2007) 7:403-8. doi:10.1126/science.113736810.1021/ nl062635n

20. Schurig D, Mock JJ, Justice BJ, Cummer SA, Pendry JB, Starr AF, et al. Metamaterial electromagnetic cloak at microwave frequencies. Science (2006) 314:977-80. doi:10.1126/science.1133628

21. Zhang FL, Li C, Fan YC, Yang RS, Shen NH, Fu QH, et al. Phase-modulated scattering manipulation for exterior cloaking in metal-dielectric hybrid metamaterials. Adv Mater (2019) 31:1903206. doi:10.1002/adma.201903206

22. Zhou L, Li H, Qin Y, Wei Z, Chan CT, Directive emissions from subwavelength metamaterial-based cavities. Appl Phys Lett (2005) 86:101101. doi:10.1063/1. 1881797
23. Li H, Hao J, Zhou L, Wei Z, Gong L, Chen H, et al. All-dimensional subwavelength cavities made with metamaterials. Appl Phys Lett (2006) 89: 104101. doi:10.1063/1.2338795

24. Ourir A, de Lustrac A, Lourtioz J-M, All-metamaterial-based subwavelength cavities (N60) for ultrathin directive antennas. Appl Phys Lett (2006) 88: 084103. doi:10.1063/1.2172740

25. Fan Y, Zhang F, Shen N-H, Fu Q, Wei Z, Li H, et al. Achieving a high-Q response in metamaterials by manipulating the toroidal excitations. Phys Rev A (2018) 97:033816. doi:10.1103/PhysRevA.97.033816

26. Zhu W, Yang R, Fan Y, Fu Q, Wu H, Zhang P, et al. Controlling optical polarization conversion with Ge2Sb2Te5-based phase-change dielectric metamaterials. Nanoscale (2018) 10:12054-61. doi:10.1039/C8NR02587H

27. Fan Y, Tu L, Zhang F, Fu Q, Zhang Z, Wei Z, et al. Broadband terahertz absorption in graphene-embedded photonic crystals. Plasmonics (2017) 13: 1153-8. doi:10.1007/s11468-017-0615-0

28. Luo M, Zhou Y, Wu S, Chen L, Wide-angle broadband absorber based on onedimensional metasurface in the visible region. Appl Phys Express (2017) 10: 092601. doi:10.7567/apex.10.092601

29. Qian Q, Sun T, Yan Y, Wang C, Large-area wide-incident-angle metasurface perfect absorber in total visible band based on coupled mie resonances. $A d v$ Opt Mater (2017) 5:1700064. doi:10.1002/adom.201700064

30. Yang F, Fan Y, Yang R, Xu J, Fu Q, Zhang F, et al. Controllable coherent perfect absorber made of liquid metal-based metasurface. Opt Express (2019) 27: 25974-82. doi:10.1364/Oe.27.025974

31. Soukoulis CM, Wegener M, Past achievements and future challenges in the development of three-dimensional photonic metamaterials. Nat Photonics (2011) 5:523-30. doi:10.1038/nphoton.2011.154

32. Sun X, Fu Q, Fan Y, Wu H, Qiu K, Yang R, et al. Thermally controllable Mie resonances in a water-based metamaterial. Sci Rep (2019) 9:5417. doi:10.1038/ s41598-019-41681-5

33. Xu J, Fan Y, Yang R, Fu Q, Zhang F, Realization of switchable EIT metamaterial by exploiting fluidity of liquid metal. Opt Express (2019) 27: 2837-43. doi:10.1364/OE.27.002837

34. Yang RS, Fu QH, Fan YC, Cai WQ, Qiu KP, Zhang WH, et al. Active control of EIT-like response in a symmetry-broken metasurface with orthogonal electric dipolar resonators. Photon Res (2019) 7:955-60. doi:10.1364/Prj.7. 000955

35. Lou J, Liang J, Yu Y, Ma H, Yang R, Fan Y, et al. Silicon-based terahertz metadevices for electrical modulation of fano resonance and transmission amplitude. Adv Opt Mater (2020) 8:2000449. doi:10.1002/adom.202000449

36. Gil I, Garcia-Garcia J, Bonache J, Martin F, Sorolla M, Marques R, Varistorloaded split ring resonators for tunable notch filters at microwave frequencies. Electron Lett (2004) 40:1347-8. doi:10.1049/el:20046389

37. Chen H, Wu B-I, Ran L, Grzegorczyk TM, Kong JA, Controllable left-handed metamaterial and its application to a steerable antenna. Appl Phys Lett (2006) 89:053509. doi:10.1063/1.2335382

38. Shadrivov IV, Morrison SK, Kivshar YS, Tunable split-ring resonators for nonlinear negative-index metamaterials. Opt Express (2006) 14:9344-9. doi:10. 1364/oe.14.009344

39. Degiron A, Mock JJ, Smith DR, Modulating and tuning the response of metamaterials at the unit cell level. Opt Express (2007) 15:1115-27. doi:10. 1364/oe.15.001115

40. Carbonell J, Boria VE, Lippens D, Nonlinear effects in split ring resonators loaded with heterostructure barrier varistors. Microw Opt Technol Lett (2008) 50:474-9. doi:10.1002/mop.23122

41. Chen H-T, O'Hara JF, Azad AK, Taylor AJ, Averitt RD, Shrekenhamer DB, et al. Experimental demonstration of frequency-agile terahertz metamaterials. Nat Photon (2008) 2:295-8. doi:10.1038/nphoton.2008.52

42. Gorkunov MV, Osipov MA, Tunability of wire-grid metamaterial immersed into nematic liquid crystal. J Appl Phys (2008) 103:036101. doi:10.1063/1. 2837099

43. Zhu W, Fan Y, Li C, Yang R, Yan S, Fu Q, et al. Realization of a near-infrared active Fano-resonant asymmetric metasurface by precisely controlling the phase transition of Ge2Sb2Te5. Nanoscale (2020) 12:8758-67. doi:10.1039/ C9NR09889E

44. Fan Y, Shen N-H, Zhang F, Wei Z, Li H, Zhao Q, et al. Electrically tunable goos-hänchen effect with graphene in the terahertz regime. Adv Opt Mater (2016) 4:1824-8. doi:10.1002/adom.201600303 
45. Fan Y, Shen N-H, Zhang F, Zhao Q, Wei Z, Zhang P, et al. Photoexcited graphene metasurfaces: significantly enhanced and tunable magnetic resonances. ACS Photon (2018) 5:1612-8. doi:10.1021/acsphotonics.8b00057

46. Fan YC, Shen NH, Zhang FL, Zhao Q, Wu HJ, Fu QH, et al. Graphene plasmonics: a platform for 2D optics. Adv Opt Mater (2019) 7:1800537. doi:10. 1002/adom.201800537

47. Khoo IC, Werner DH, Liang X, Diaz A, Weiner B, Nanosphere dispersed liquid crystals for tunable negative-zero-positive index of refraction in the optical and terahertz regimes. Opt Lett (2006) 31:2592-4. doi:10.1364/OL.31. 002592

48. Wangberg R, Elser J, Narimanov EE, Podolskiy VA, Nonmagnetic nanocomposites for optical and infrared negative-refractive-index media. J Opt Soc Am B (2006) 23:498-505. doi:10.1364/josab.23.000498

49. Wang X, Kwon DH, Werner DH, Khoo IC, Kildishev AV, Shalaev VM, Tunable optical negative-index metamaterials employing anisotropic liquid crystals. Appl Phys Lett (2007) 91:143122. doi:10.1063/1.2795345

50. Werner DH, Kwon DH, Khoo IC, Kildishev AV, Shalaev VM, Liquid crystal clad near-infrared metamaterials with tunable negative-zero-positive refractive indices. Opt Express (2007) 15:3342-7. doi:10.1364/oe.15.003342

51. Khoo IC, Nonlinear optics, active plasmonics and metamaterials with liquid crystals. Prog Quantum Electron (2014) 38:77-117. doi:10.1016/j.pquantelec. 2014.03.001

52. Zhang W, Song QH, Zhu WM, Shen ZX, Chong P, Tsai DP, et al. Metafluidic metamaterial: a review. Adv Phys X (2018) 3:20. doi:10.1080/23746149.2017. 1417055

53. Zhao Q, Kang L, Du B, Li B, Zhou J, Tang H, et al. Electrically tunable negative permeability metamaterials based on nematic liquid crystals. Appl Phys Lett (2007) 90:011112. doi:10.1063/1.2430485

54. Zhang F, Zhao Q, Kang L, Gaillot DP, Zhao X, Zhou J, et al. Magnetic control of negative permeability metamaterials based on liquid crystals. Appl Phys Lett (2008) 92:193104. doi:10.1063/1.2926678

55. Zhang FL, Zhao Q, Gaillot DP, Zhao XP, Lippens D, Numerical investigation of metamaterials infiltrated by liquid crystal. J Opt Soc Am B (2008) 25:1920-5. doi:10.1364/josab.25.001920

56. Khoo IC, Nonlinear optics of liquid crystalline materials. Phys Rep (2009) 471: 221-67. doi:10.1016/j.physrep.2009.01.001

57. Xiao S, Chettiar UK, Kildishev AV, Drachev V, Khoo IC, Shalaev VM, Tunable magnetic response of metamaterials. Appl Phys Lett (2009) 95:033115. doi:10. $1063 / 1.3182857$

58. Zhang F, Kang L, Zhao Q, Zhou J, Zhao X, Lippens D, Magnetically tunable left handed metamaterials by liquid crystal orientation. Opt Express (2009) 17: 4360-6. doi:10.1364/oe.17.004360

59. Zhang F, Zhao Q, Zhang W, Sun J, Zhou J, Lippens D, Voltage tunable short wire-pair type of metamaterial infiltrated by nematic liquid crystal. Appl Phys Lett (2010) 97:134103. doi:10.1063/1.3496034

60. Zhang F, Zhang W, Zhao Q, Sun J, Qiu K, Zhou J, et al. Electrically controllable fishnet metamaterial based on nematic liquid crystal. Opt Express (2011) 19: 1563-8. doi:10.1364/oe.19.001563

61. Vasić B, Zografopoulos DC, Isić G, Beccherelli R, Gajić R, Electrically tunable terahertz polarization converter based on overcoupled metal-isolator-metal metamaterials infiltrated with liquid crystals. Nanotechnology (2017) 28: 124002. doi:10.1088/1361-6528/aa5bbd

62. Isić G, Vasić B, Zografopoulos DC, Beccherelli R, Gajić R, Electrically tunable critically coupled terahertz metamaterial absorber based on nematic liquid crystals. Phys Rev Appl (2015) 3:064007. doi:10.1103/PhysRevApplied.3. 064007

63. Wang J, Tian H, Wang Y, Li X, Cao Y, Li L, et al. Liquid crystal terahertz modulator with plasmon-induced transparency metamaterial. Opt Express (2018) 26:5769-76. doi:10.1364/oe.26.005769

64. Lu H, Jing S, Xia T, Yang J, Yin Z, Deng G, Measurement of LC dielectric constant at lower terahertz region based on metamaterial absorber. IEICE Electron Express (2017) 14:20170469. doi:10.1587/elex.14.20170469

65. Wang PY, Jin T, Meng FY, Lyu YL, Erni D, Wu Q, et al. Numerical investigation of nematic liquid crystals in the $\mathrm{THz}$ band based on EIT sensor. Opt Express (2018) 26:12318-29. doi:10.1364/oe.26.012318

66. Yin Z, Lu Y, Xia T, Lai W, Yang J, Lu H, et al. Electrically tunable terahertz dual-band metamaterial absorber based on a liquid crystal. RSC Adv (2018) 8: 4197-203. doi:10.1039/C7RA13047C
67. Shen ZX, Zhou SH, Ge SJ, Hu W, Lu YQ, Liquid crystal enabled dynamic cloaking of terahertz Fano resonators. Appl Phys Lett (2019) 114:5. doi:10. 1063/1.5082224

68. Ji Y, Fan F, Zhang X, Cheng J, Chang S, Active terahertz anisotropy and dispersion engineering based on dual-frequency liquid crystal and dielectric metasurface. J Light Technol (2020) 38:4030-6. doi:10.1109/jlt.2020.2985667

69. Wu JB, Shen Z, Ge SJ, Chen BW, Shen ZX, Wang TF, et al. Liquid crystal programmable metasurface for terahertz beam steering. Appl Phys Lett (2020) 116:5. doi:10.1063/1.5144858

70. Shrekenhamer D, Chen WC, Padilla WJ, Liquid crystal tunable metamaterial absorber. Phys Rev Lett (2013) 110:177403. doi:10.1103/PhysRevLett.110. 177403

71. Savo S, Shrekenhamer D, Padilla WJ, Liquid crystal metamaterial absorber spatial light modulator for THz applications. Adv Opt Mater (2014a) 2:275-9. doi:10.1002/adom.201300384

72. Yang L, Fan F, Chen M, Zhang X, Chang S-J, Active terahertz metamaterials based on liquid-crystal induced transparency and absorption. Opt Commun (2017) 382:42-8. doi:10.1016/j.optcom.2016.07.055

73. Shen Z, Zhou S, Ge S, Duan W, Chen P, Wang L, et al. Liquid-crystalintegrated metadevice: towards active multifunctional terahertz wave manipulations. Opt Express (2018) 43:4695-8. doi:10.1364/ol.43.004695

74. Ha NY, Jeong SM, Nishimura S, Takezoe H, Color-temperature tunable white reflector using bichiral liquid crystal films. Opt Express (2010) 18:26339-44. doi:10.1364/oe.18.026339

75. Khoo IC, Diaz A, Liou J, Stinger MV, Huang J, Ma Y, Liquid crystals tunable optical metamaterials. IEEE J Sel Top Quantum Electron (2010) 16:410-7. doi:10.1109/jstqe.2009.2032246

76. Liu L, Shadrivov IV, Powell DA, Raihan MR, Hattori HT, Decker M, et al. Temperature control of terahertz metamaterials with liquid crystals. IEEE Trans Terahertz Sci Technol (2013) 3:827-31. doi:10.1109/tthz.2013.2285570

77. Kowerdziej R, Olifierczuk M, Parka J, Wrobel J, Terahertz characterization of tunable metamaterial based on electrically controlled nematic liquid crystal. Appl Phys Lett (2014) 105:022908. doi:10.1063/1.4890850

78. Maasch M, Roig M, Damm C, Jakoby R, Voltage-tunable artificial gradientindex lens based on a liquid crystal loaded fishnet metamaterial. IEEE Antennas Wirel Propag Lett (2014) 13:1581-4. doi:10.1109/lawp.2014.2345841

79. Chen CC, Chiang WF, Tsai MC, Jiang SA, Chang TH, Wang SH, et al Continuously tunable and fast-response terahertz metamaterials using inplane-switching dual-frequency liquid crystal cells. Opt Lett (2015) 40:2021-4. doi:10.1364/ol.40.002021

80. Zografopoulos DC, Beccherelli R, Tunable terahertz fishnet metamaterials based on thin nematic liquid crystal layers for fast switching. Sci Rep (2015) 5: 13137. doi:10.1038/srep13137

81. Komar A, Fang Z, Bohn J, Sautter J, Decker M, Miroshnichenko A, et al. Electrically tunable all-dielectric optical metasurfaces based on liquid crystals. Appl Phys Lett (2017) 110:4. doi:10.1063/1.4976504

82. Yang J, Wang P, Shi T, Gao S, Lu H, Yin Z, et al. Electrically tunable liquid crystal terahertz device based on double-layer plasmonic metamaterial. Opt Express (2019) 27:27039-45. doi:10.1364/OE.27.027039

83. Zou CJ, Komar A, Fasold S, Bohn J, Muravsky AA, Murauski AA, et al. Electrically tunable transparent displays for visible light based on dielectric metasurfaces. ACS Photonics (2019) 6:1533-40. doi:10.1021/acsphotonics. $9 \mathrm{~b} 00301$

84. Bhardwaj A, Sridurai V, Puthoor NM, Nair AB, Ahuja T, Nair GG, Evidence of tunable fano resonance in a liquid crystal-based colloidal metamaterial. $A d v$ Opt Mater (2020) 8:1901842. doi:10.1002/adom.201901842

85. Liu Y, Song J, Zhao W, Ren X, Cheng Q, Luo X, et al. Dynamic thermal camouflage via a liquid-crystal-based radiative metasurface. Nanophotonics (2020) 9:855-63. doi:10.1515/nanoph-2019-0485

86. Sandford O'Neill JJ, Salter PS, Booth MJ, Elston SJ, Morris SM, Electricallytunable positioning of topological defects in liquid crystals. Nat Commun (2020) 11:2203. doi:10.1038/s41467-020-16059-1

87. Kossyrev PA, Yin A, Cloutier SG, Cardimona DA, Huang D, Alsing PM, et al. Electric field tuning of plasmonic response of nanodot array in liquid crystal matrix. Nano Lett (2005) 5:1978-81. doi:10.1021/nl0513535

88. Yaghmaee P, Karabey OH, Bates B, Fumeaux C, Jakoby R, Electrically tuned microwave devices using liquid crystal technology. Int J Antennas Propag (2013) 2013:9. doi:10.1155/2013/824214 
89. Buchnev O, Podoliak N, Kaczmarek M, Zheludev NI, Fedotov VA, Electrically controlled nanostructured metasurface loaded with liquid crystal: toward multifunctional photonic switch. Adv Opt Mater (2015) 3:674-9. doi:10. 1002/adom.201400494

90. Lee YU, Kim J, Wu JW, Electro-optic switching in metamaterial by liquid crystal. Nano Converg (2015) 2:23. doi:10.1186/s40580-015-0054-6

91. Chen KP, Ye SC, Yang CY, Yang ZH, Lee W, Sun MG, Electrically tunable transmission of gold binary-grating metasurfaces integrated with liquid crystals. Opt Express (2016) 24:16815-21. doi:10.1364/OE.24.016815

92. Lagerwall JPF, Scalia G, A new era for liquid crystal research: applications of liquid crystals in soft matter nano-, bio- and microtechnology. Curr Appl Phys (2012) 12:1387-412. doi:10.1016/j.cap.2012.03.019

93. Khoo IC, DC-field-assisted grating formation and nonlinear diffractions in methyl-red dye-doped blue phase liquid crystals. Opt Lett (2015) 40:60-3. doi:10.1364/OL.40.000060

94. Giden IH, Eti N, Rezaei B, Kurt H, Adaptive graded index photonic crystal lens design via nematic liquid crystals. IEEE J Quantum Electron (2016) 52:7. doi:10.1109/jqe.2016.2605398

95. Ho TJ, Chen CW, Khoo IC, Polarization-free and high-resolution holographic grating recording and optical phase conjugation with azo-dye doped bluephase liquid crystals. Liq Cryst (2018) 45:1944-52. doi:10.1080/02678292.2018. 1491068

96. Vaskin A, Bohn J, Chong KE, Bucher T, Zilk M, Choi D-Y, et al. Directional and spectral shaping of light emission with mie-resonant silicon nanoantenna arrays. ACS Photonics (2018) 5:1359-64. doi:10.1021/acsphotonics.7b01375

97. Guo DY, Chen CW, Li CC, Jau HC, Lin KH, Feng TM, et al. Reconfiguration of three-dimensional liquid-crystalline photonic crystals by electrostriction. Nat Mater (2020) 19:94-101. doi:10.1038/s41563-019-0512-3

98. Buchnev O, Wallauer J, Walther M, Kaczmarek M, Zheludev NI, Fedotov VA, Controlling intensity and phase of terahertz radiation with an optically thin liquid crystal-loaded metamaterial. Appl Phys Lett (2013) 103:4. doi:10.1063/1. 4823822

99. Chikhi N, Lisitskiy M, Papari G, Tkachenko V, Andreone A, A hybrid tunable THz metadevice using a high birefringence liquid crystal. Sci Rep (2016) 6: 34536. doi:10.1038/srep34536

100. Sautter J, Staude I, Decker M, Rusak E, Neshev DN, Brener I, et al. Active tuning of all-dielectric metasurfaces. ACS Nano (2015) 9:4308-15. doi:10. 1021/acsnano. 5 b00723

101. Liang X, Chen M, Guo S, Zhang L, Li F, Yang H, Dual-band modulation of visible and near-infrared light transmittance in an all-solution-processed hybrid micro-nano composite film. ACS Appl Mater Interf (2017) 9:40810-9. doi:10.1021/acsami.7b11582

102. Parry M, Komar A, Hopkins B, Campione S, Liu S, Miroshnichenko AE, et al. Active tuning of high-Q dielectric metasurfaces. Appl Phys Lett (2017) 111: 053102. doi:10.1063/1.4997301

103. Bohn J, Bucher T, Chong KE, Komar A, Choi DY, Neshev DN, et al. Active tuning of spontaneous emission by mie-resonant dielectric metasurfaces. Nano Lett (2018) 18:3461-5. doi:10.1021/acs.nanolett.8b00475

104. Ke YJ, Zhou CZ, Zhou Y, Wang SC, Chan SH, Long Y, Emerging thermalresponsive materials and integrated techniques targeting the energy-efficient smart window application. Adv Funct Mater (2018) 28:18. doi:10.1002/adfm. 201800113

105. Sentker K, Yildirim A, Lippmann M, Zantop AW, Bertram F, Hofmann T, et al. Self-assembly of liquid crystals in nanoporous solids for adaptive photonic metamaterials. Nanoscale (2019) 11:23304-17. doi:10.1039/ c9nr07143a

106. Hokmabadi MP, Tareki A, Rivera E, Kung P, Lindquist RG, Kim SM, Investigation of tunable terahertz metamaterial perfect absorber with anisotropic dielectric liquid crystal. $A I P A d v$ (2017) 7: 015102. doi:10. $1063 / 1.4973638$

107. Yin ST, Xiao D, Liu JX, Li K, He H, Jiang SZ, et al. Reconfigurable chiral metasurface absorbers based on liquid crystals. IEEE Phot J (2018) 10:9. doi:10.1109/jphot.2018.2878775

108. Vasic B, Zografopoulos DC, Isic G, Beccherelli R, Gajic R, Electrically tunable terahertz polarization converter based on overcoupled metal-isolator-metal metamaterials infiltrated with liquid crystals. Nanotechnology (2017) 28:11. doi:10.1088/1361-6528/aa5bbd

109. Sasaki T, Nishie Y, Kambayashi M, Sakamoto M, Noda K, Okamoto H, et al. Active terahertz polarization converter using a liquid crystal-embedded metal mesh. IEEE Phot J (2019) 11:1-7. doi:10.1109/jphot.2019.2950021

110. Savo S, Shrekenhamer D, Padilla WJ, Liquid crystal metamaterial absorber spatial light modulator for THz applications. Adv Opt Mater (2014b) 2:275-9. doi:10.1002/adom.201300384

111. Zeng BB, Huang ZQ, Singh A, Yao Y, Azad AK, Mohite AD, et al. Hybrid graphene metasurfaces for high-speed mid-infrared light modulation and single-pixel imaging. Light Sci Appl (2018) 7:51. doi:10.1038/s41377-0180055-4

112. Ting TL, Technology of liquid crystal based antenna [Invited]. Opt Express (2019) 27:17138-53. doi:10.1364/oe.27.017138

113. Sharma M, Hendler N, Ellenbogen T, Electrically switchable color tags based on active liquid-crystal plasmonic metasurface platform. Adv Opt Mater (2020) 8:1901182. doi:10.1002/adom.201901182

114. Buchnev O, Ou JY, Kaczmarek M, Zheludev NI, Fedotov VA, Electro-optical control in a plasmonic metamaterial hybridised with a liquid-crystal cell. Opt Express (2013) 21:1633-8. doi:10.1364/oe.21.001633

115. Decker M, Kremers C, Minovich A, Staude I, Miroshnichenko AE, Chigrin D, et al. Electro-optical switching by liquid-crystal controlled metasurfaces. Opt Express (2013) 21:8879-85. doi:10.1364/oe.21.008879

Conflict of Interest: The authors declare that the research was conducted in the absence of any commercial or financial relationships that could be construed as a potential conflict of interest.

Copyright (๑) $2021 \mathrm{Xu}$, Yang, Fan, Fu and Zhang. This is an open-access article distributed under the terms of the Creative Commons Attribution License (CC BY). The use, distribution or reproduction in other forums is permitted, provided the original author(s) and the copyright owner(s) are credited and that the original publication in this journal is cited, in accordance with accepted academic practice. No use, distribution or reproduction is permitted which does not comply with these terms. 